\title{
La Deutsche Gesellschaft für Erforschung des 18. Jahrhunderts
}

\section{Guillaume Garner}

\section{OpenEdition}

\section{Journals}

Édition électronique

URL : http://journals.openedition.org/ifha/354

DOI : $10.4000 /$ ifha.354

ISSN : 2198-8943

\section{Éditeur}

IFRA - Institut franco-allemand (sciences historiques et sociales)

\section{Édition imprimée}

Date de publication : 30 septembre 2009

Pagination : 171-174

ISSN : 2190-0078

\section{Référence électronique}

Guillaume Garner, "La Deutsche Gesellschaft für Erforschung des 18. Jahrhunderts », Revue de l'IFHA [En ligne], 1 | 2009, mis en ligne le 07 février 2013, consulté le 21 avril 2019. URL : http:// journals.openedition.org/ifha/354; DOI : 10.4000/ifha.354

Ce document a été généré automatiquement le 21 avril 2019.

(CIFHA 


\title{
La Deutsche Gesellschaft für Erforschung des 18. Jahrhunderts
}

\author{
Guillaume Garner
}

1 En raison notamment de la richesse de ses fonds, la Herzog August Bibliothek (HAB) de Wolfenbüttel est devenue depuis une quarantaine d'années un lieu incontournable de la recherche sur les trois siècles de la période moderne. C'est pour cette raison que de nombreuses institutions de recherche dont la Société allemande de recherche sur le XVIIIe siècle/ Deutsche Gesellschaft für Erforschung des 18. Jahrhunderts (DGEJ) sont rattachées à cette prestigieuse institution.

2 La DGEJ a été fondée en 1975, à une époque où les chercheurs spécialistes de l'Aufklärung étaient relativement moins nombreux qu'aujourd'hui et se trouvaient surtout dans état de un relatif isolement, ne bénéficiant pas d'institution susceptible de fournir un lieu de rencontre et d'échange comme il en existait dans les pays anglo-saxons, en France ou en RDA où existaient deux institutions particulièrement dynamiques, localisées à Halle et à Potsdam. Les fondateurs de la DGEJ souhaitaient donc d'une part constituer un réseau réunissant les chercheurs (ouest-)allemands, d'autre part renforcer la visibilité des recherches menées en Allemagne sur l'Aufklärung à l'échelle internationale. Cette fondation est d'ailleurs intervenue à un moment où la période moderne était reconnue comme une unité historique possédant ses spécificités propres (comme le montre par exemple l'essor des chaires de Frühe Neuzeit dans les universités) ${ }^{1}$ et où l'intérêt pour l' Aufklärung allait croissant. À une époque où les problématiques de la modernisation et d'un éventuel Sonderweg allemand guidaient nombre de recherches, il s'agissait en effet de s'interroger à la fois sur les éventuelles spécificités de l'Aufklärung allemande par rapport au mouvement des Lumières dans d'autres pays, et sur les raisons pour lesquelles l' Aufklärung avait été l'objet, entre 1770 et 1830 , de jugements de plus en plus négatifs ${ }^{2}$. Nulle surprise donc de constater que l'un des principaux historiens promoteurs de l' Aufklärungforschung en histoire, Rudolf Vierhaus, ait compté parmi les membres fondateurs de la DGEJ. 
3 Les activités de la DGEJ ont, dès le départ, été marquées par trois principales positions de principe : l'interdisciplinarité, le comparatisme, et la volonté de considérer l'Aufklärung au sens large, c'est-à-dire comme une période, en d'autres termes la volonté d'étudier le XVIIIe siècle dans tous ses aspects.

4 Cette institution compte actuellement plus de 850 membres en Allemagne et dans de nombreux pays étrangers, et s'est imposée comme l'un des acteurs de majeurs de l' Aufklärungsforschung, grâce à des activités diversifiées.

5 La DGEJ organise d'abord à un rythme annuel des manifestations scientifiques parmi lesquels il convient de distinguer les colloques spécifiques de la société et des colloques trilatéraux, organisés conjointement avec des institutions françaises et italiennes.

6 Les premiers sont parfois intégralement pris en charge par la DGEJ, comme ce fut le cas en 2006 du colloque sur « les cultures du savoir », parfois organisés en collaboration avec des centres de recherches également spécialisés dans la recherche sur le XVIIIe siècle : le colloque «Die Sachen der Aufklärung - Matters of Enlightenment - La cause et les choses des Lumières » qui se tiendra en octobre 2010 est ainsi organisé par la DGEJ en collaboration avec le Centre Interdisciplinaire de Recherches sur les Lumières en Europe (IZEA) de Halle, tout comme le colloque de 2008 sur « l'Europe et la Turquie au XVIIIe siècle » avait été organisé en collaboration avec la Faculté de Philosophie de l'Université de Bonn.

7 Les colloques trilatéraux (franco-italo-allemands) sont plus récents, le premier s'étant tenu en 2007 à Potsdam («Aufklärung - Lumières - Illuminismo »), et il fut suivi l'année suivante d'un colloque à Metz sur la « Gallophilie et gallophobie dans la littérature et les médias en Allemagne et en Italie au XVIIIe siècle ", tandis que le colloque d'octobre 2009, portant sur «les tropes et les métaphores dans le discours des sciences humaines ( Geisteswissenschaften) au XVIIIe siècle ", s'est déroulé à l'Université de Bergame.

Comme on le voit à travers les partenariats à l'origine de ces colloques, la DGEJ joue un rôle important de mise en relation d'institutions diverses. Ce rôle est matérialisé par un autre projet, à savoir l'élaboration d'une "topographie de la recherche sur le XVIIIe siècle " (Topographie der 18. Jahrhundert-Forschung) : il s'agit d'un répertoire inventoriant les institutions universitaires et extra-universitaires allemandes (sociétés savantes, archives, musées, etc.) travaillant sur le XVIIIe siècle. Ce répertoire organisé par ordre alphabétique est en cours de constitution, sur la base de déclarations des institutions souhaitant y figurer, et il peut être consulté sur le site internet de la DGEJ ${ }^{3}$.

Un autre point fort de la DGEJ concerne son activité éditoriale. Les publications éditées par la société sont de deux types principaux, mais présentent toutes des points communs : comparatisme - l'espace allemand est loin d'être le seul à être traité - et interdisciplinarité, puisque collaborent à ces publications historiens, philosophes, germanistes, historiens des sciences ou spécialistes des études littéraires. Il s'agit d'une part de revues scientifiques. La principale est propre à la DGEJ : la revue semestrielle Das achtzehnte Jahrhundert. Fondée en 1977, elle comprend dans une première partie des articles, qui parfois portent sur des thèmes variés, parfois sont regroupés autour d'un thème : des dossiers ont ainsi ces dernières années été consacrées à des thèmes aussi divers que "culture et économie» (2008), "le pyrrhonisme historique» (2007), les concepts du temps au XVIIIe siècle (2006) ou les rapports entre "genre littéraire et genre » (Gattung und Geschlecht) en 2005. La seconde partie est consacrée aux comptes rendus d'ouvrages (environ une quinzaine par numéro). La seconde revue, dont la DGEJ est coéditrice, s'intitule Aufklärung. À l'origine semestrielle, annuelle depuis 2001, cette 
revue consacre chacun de ses numéros à un thème précis - citons à titre d'exemple : «John Locke. Aspects de sa philosophie pratique et théorique » (2006), la « Frühaufklärung "(2005) ou "Aufklärung et anthropologie au XVIIIe siècle» (2002). Alors que Das achtzehnte Jahrhundert est tournée principalement vers des thématiques d'histoire culturelle, la revue Aufklärung est, quant à elle, plutôt centrée sur des questions d'histoire des idées et d'histoire de la philosophie.

Outre ces périodiques, la DGEJ édite également deux collections d'ouvrages. La première, intitulée «Das achtzehnte Jahrhundert. Supplementa », publie principalement des ouvrages d'histoire culturelle, dont certains sont des recueils issus de colloques organisés par la société comme c'est par exemple le cas de l'ouvrage sur le tremblement de terre de Lisbonne et le discours sur les catastrophes en Europe au XVIIIe siècle ${ }^{4}$. La seconde collection, les "Studien zum 18. Jahrhundert », est consacrée à la publication d'ouvrages ou de recueils consacrés à l'histoire intellectuelle et à l'histoire des idées, et a publié des livres devenus des œuvres de référence pour les historiens des Lumières.

\section{ANNEXES}

Deutsche Gesellschaft für Erforschung des 18. Jahrhunderts

Herzog August Bibliothek

Postfach 1364

D-38299 Wolfenbüttel

PD Dr. Ulrike Zeuch

Tel. (0049)/0/5331 808-202

Regina Zimpel

Tel. (0049) (0)5331 808-203

Fax : (0049) (0)5331 808-266

Site internet : http://dgej.hab.de

Adresse électronique : dgej@hab.de

\section{NOTES}

1. Winfried Schulze, «L'époque moderne /Frühe Neuzeit) entre expérience individuelle et approche structurelle : bilan, déficits et concepts d'un champ de recherches » in : BullMHFA, 41, 2005, p. 252-281.

2. Voir les réflexions de Rudolf Vierhaus, «Die Erforschung des 18. Jahrhunderts. Aufklärungsaktivitäten - Desiderate - Defizite » in: Das achtzehnte Jahrhundert, 19, 1995, p. 158-162.

3. Voir le lien : http://dgej.hab.de/topographie.htm.

4. Voir compte rendu dans ce numéro, p. 442. 


\section{AUTEUR}

\section{GUILLAUME GARNER}

Guillaume Garner est chargé des études modernes à la MHFA / IFHA depuis 2006. 